\title{
O hospital e sua centralidade no complexo industrial da saúde
}

\author{
The central role of Hospitals to the health industrial complex
}

\author{
Fernando Diório Alves dos Santos \\ Mestre em Geografia Humana pela \\ Universidade de São Paulo \\ fernando.diorio@usp.br
}

\author{
Tatiana dos Santos Thomaz \\ Mestranda em Geografia Humana pela \\ Universidade de São Paulo \\ tatiana.thomaz@usp.br
}

Resumo: A economia da saúde, composta pela demanda social de bens e serviços, pela formação de profissionais, por indústrias de alta tecnologia e por produção e consumo de insumos e medicamentos, pode ser entendida por meio do atual paradigma da prática médica, a medicina tecnológica, e o conceito de complexo industrial da saúde. Propomos nesse artigo um olhar ao hospital, sistema técnico capaz de orientar e dinamizar os fluxos materiais e imateriais ligados à saúde e à prática médica, destacando a centralidade dos conjuntos formados pela Escola Paulista de Medicina da Universidade Federal de São Paulo/Hospital São Paulo e a Faculdade de Medicina da Universidade de São Paulo/Hospital das Clínicas.

Palavras-chave: Complexo Industrial da Saúde, Hospital das Clínicas, Hospital São Paulo.

\begin{abstract}
Comprised by social demand for goods and services, specific professional education, cutting-edge technology industries and the production of medicines and intermediary goods, health economy can be understood through the current paradigm in medical practice, the technologic medicine, and through the concept of health industrial complex. In this paper, hospitals will be envisioned as sophisticated technical systems able to guide and increase the dynamics of material and immaterial flows related to medical practice and health economy. These theoretical remarks and the central role developed by hospital in the health economy will be empirically illustrated by the cases of two hospital complexes: Escola Paulista de Medicina da Universidade Federal de São Paulo/Hospital São Paulo and the Faculdade de Medicina da Universidade de São Paulo/Hospital das Clínicas.
\end{abstract}

Keywords: Health Industrial Complex, Clinicas Hospital, Hospital São Paulo. 


\section{Introdução}

Tratamos a saúde aqui ${ }^{1}$ como área importante de inovação, investimentos, renda e emprego, que se compõe, de um lado, pela demanda social por bens e serviços de saúde e, de outro, pela formação de profissionais, por indústrias, por prestadores de serviços médicos, pela produção e consumo de insumos e medicamentos (GADELHA, 2003; GADELHA, QUENTAL \& FIALHO, 2003). Em ambos os casos, atua entre a racionalidade técnica e a racionalidade política: o perfil do gasto público e sua relação com o Produto Interno Bruto fomenta o desenvolvimento dessa racionalidade técnica segundo um padrão de desenvolvimento econômico e as formas de organização e desempenho das unidades produtoras de saúde; a racionalidade política da saúde se expressa como direito, como bem econômico e como campo de acumulação de capital (VIANA, SILVA \& ELIAS, 2005), com “o objetivo de formular novas balizas na conceituação da sociedade vigente, enquadrando e controlando o homem e seu corpo a partir de critérios que lhe designariam um estado normal ou patológico de 'estar no mundo"" (MOTA, 2005:19).

O desenvolvimento de um novo paradigma na prática médica - a medicina tecnológica - e a institucionalização do chamado complexo industrial da saúde como norte para as decisões estratégicas do Estado e do capital privado apresentam estreita interação entre ciência, técnica e informação atribuindo o caráter de mercadoria à saúde e tornando-a um campo de acumulação de capital, sendo a operacionalização dos conceitos de circuito espacial produtivo e círculos de cooperação no espaço importantes para compreender a espacialidade desse novo paradigma.

Reforçamos como um elemento importante para a atual medicina, elo entre a produção industrial e científica promovida pelo complexo industrial da saúde e o atendimento à população, o hospital - instituição participante da dinâmica da economia

\footnotetext{
${ }^{1}$ A pesquisa apresentada nesse artigo faz parte da investigação conduzida pelo Prof. Dr. Ricardo Mendes Antas Jr., "Reestruturação urbana e refuncionalizações do espaço: o complexo industrial da saúde no estado de São Paulo e suas relações com a urbanização contemporânea", com a qual dialogam as pesquisas de mestrado: de Mait Bertollo, "O circuito espacial produtivo da vacina no território brasileiro e a pandemia Influenza A H1N1"; de Rafael da Silva Almeida, "Rede urbana no estado de São Paulo e serviços de análises laboratoriais de saúde: o circuito espacial produtivo dos reagentes para diagnóstico"; de Flávio de Campos Vendrúsculo, "As feiras e congressos médicos como círculos de cooperação no espaço: a integração do complexo industrial da saúde e a inserção da lógica corporativa no hospital"; de Tatiana dos Santos Thomaz, "A conformação do complexo médico-hospitalar na cidade de São Paulo: a participação do Hospital das Clínicas (USP) e do Hospital São Paulo (Unifesp) nas transformações urbanas", e de Fernando Diório Alves dos Santos, "O circuito espacial produtivo e a topologia dos equipamentos de radiologia e diagnóstico por imagem no território brasileiro”.
} 
urbana (DAVID, 2010) e centrado na configuração dos circuitos espaciais produtivos da saúde e na acumulação ampliada de capitais, concentrando poder econômico e legitimando certa forma de cuidados médicos (ANTAS JR., 2011a).

Esse processo pode ser verificado no Hospital das Clínicas da Universidade de São Paulo e no Hospital São Paulo da Universidade Federal de São Paulo, ambos de alta complexidade com ensino e pesquisas médico-científicas, constituindo-se elementos centrais no complexo industrial da saúde, bem como para o desenvolvimento dos circuitos espaciais produtivos da saúde e seus respectivos círculos de cooperação no espaço (SANTOS \& SILVEIRA, 2001; SANTOS, 2012) capazes de promover refuncionalizações no espaço (ANTAS JR., 2011b).

\section{O atual paradigma da prática médica: a medicina tecnológica}

Destacamos a transformação do trabalho médico apontada por Lilia Schraiber, numa periodização atenta às mudanças na organização dos serviços e na base do trabalho:

[...] é possível dizer que, desde o momento em que a medicina se inscreve na ordem técnico-científica moderna, desenvolveram-se dois modos de estruturação da prática. O primeiro ocupou todo o século XIX até aproximadamente os anos 30 do século XX, começando o segundo a configurar-se no período de 1930-50, a partir do que implanta-se de forma generalizada. Essa periodização corresponde ao que ocorreu nos países que iniciaram a reestruturação da vida social conforme o modo capitalista de produção, e que só mais tardiamente se estabeleceu na sociedade brasileira (1993: 179).

Classificados respectivamente como medicina liberal e medicina tecnológica, os dois períodos transitam de uma prática médica mais artesanal, no qual o exercício pessoal do médico era imprescindível, ao emprego em grande escala de medicamentos e instrumentos diagnósticos. Ao longo dos anos, o trabalho individual submeteu-se às especialidades médicas, e a prática isolada nos consultórios perdeu espaço para o trabalho coletivo em clínicas ambulatoriais e unidades hospitalares, "a interdependência 
(ao invés da independência) e a cooperação (em substituição ao produtor isolado)" (SCHRAIBER, 1993: 172).

Jairnilson Paim (2009) reforça essa periodização do trabalho médico caracterizando a medicina liberal como o momento em que o profissional estabelecia as condições de atendimento, horário e preço das consultas em seu consultório particular empregando, quando considerasse necessário, os instrumentos de trabalho sob sua propriedade. A medicina tecnológica ou empresarial, por sua vez, reduziu essa autonomia - o controle da clientela, o estabelecimento do preço das consultas e os procedimentos - com "a ampliação da utilização de equipamentos e de meios de diagnóstico e tratamento, inclusive o aumento exponencial dos custos da assistência" (PAIM, 2009: 35).

No caso brasileiro, Schraiber (1993) assinala a década de 1960 como o ponto em que a medicina liberal é definitivamente superada, com a mudança qualitativa liderada pela criação do Instituto Nacional de Previdência Social (INPS) e a reorganização do produtor privado de serviços em moldes mais tipicamente empresariais. Nesse momento, ampliou-se a oferta de empregos no setor público (inserção no mercado de trabalho pelo assalariamento), criaram-se novas unidades hospitalares privadas (a medicina lucrativa) e se multiplicaram as unidades individuais de serviços complementares (diagnósticos ou terapêuticos) (PAIM, 2009).

Assim, a medicina tecnológica instaura novas formas de propriedade dos meios de produção, bem como a ação, o volume e o tempo gasto nas consultas:

Essa medicina tecnológica, portanto, também representará a penetração do capital privado para nela ingressar, da mesma forma como ocorre com a capitalização de outros ramos da produção, haverá, nas distintas formações sociais, modos particulares de realização do capital: maior ou menor presença do Estado como produtor direto e/ou como financiador de produtores e consumidores; maior ou menor viabilização de pequenos produtores privados, constituindo empresas médicas, independentes ou sob a intermediação de seguros-saúde privados (SCHRAIBER, 1993: 197).

As mudanças na produção social do trabalho médico implicam, simultaneamente, extensão e diferenciação dos serviços, reconhecimento extensivo do direito à saúde, universalização da assistência com elitização do consumo, progressiva 
especialização, incorporação de equipamentos, trabalho associativo, produção na forma de empresa, aumento dos custos, clientelas coletivas, setor público e privado conectados por vários e conflituosos mecanismos, complexo médico-industrial, medicina tecnologicamente dependente, produção individual dependente da gerência institucional e produção institucional dependente das políticas públicas em saúde e, por fím, institucionalização de diferenças e desigualdades na produção e no consumo (SCHRAIBER, 2008).

Com o desenvolvimento da medicina tecnológica, estabelecem-se as condições para que os equipamentos intensivos em tecnologia ganhem centralidade e determinem o trabalho médico. A partir daí se verificam as mudanças nas relações dos agentes de trabalho (segundo o custo dos equipamentos e a possibilidade de compra), a racionalidade clínica (ultratécnica e tendencialmente impessoal, respondendo a uma clínica protocolada que reduz a subjetividade do ato técnico) e a noção de saúde como objeto de consumo (bem e serviços que refletem a ideologia da saúde e da doença como fenômenos individuais e naturais) (SCHRAIBER, 1993).

Como vimos, esse paradigma é pautado na interdependência e na cooperação, com o largo emprego de medicamentos e de instrumentos diagnósticos intensivos em tecnologia, determinando a prática médica e a noção de saúde como bens de consumo condicionados ao poder de compra do cliente. Nessa perspectiva, a compreensão da chamada medicina tecnológica subsidia o contexto em que se inserem os produtos de diferentes circuitos espaciais produtivos, apresentados adiante.

\section{O complexo industrial da saúde}

Esse complexo pode ser delimitado "a partir de um conjunto selecionado de atividades produtivas que mantêm relações intersetoriais de compra e venda de bens e serviços [...] e/ou conhecimentos e tecnologias", em um "contexto político e institucional bastante particular dado pelas especificidades da área da saúde" (GADELHA, 2003: 523). É composto por três grandes grupos de atividades, congregando: (I) as indústrias de base química e biotecnológica, (II) as indústrias de base mecânica, eletrônica e de materiais e (III) setores prestadores de serviços, segmento que, para o autor, dá organicidade ao complexo e se constitui como setor 
motriz, por fazer confluir a produção dos demais a partir dos dados relativos à sua expansão ou contração.

Reiterando essas relações intersetoriais e a primazia dos serviços no complexo industrial da saúde, destacamos na figura 1 a importante relação entre as indústrias produtoras de bens (base química e biotecnológica e base mecânica, eletrônica e de materiais) e os setores prestadores de serviços, mediada pelo fornecimento de produtos e a comunicação de demandas - respostas ao uso dos produtos ou a solicitações de ordem prática que orientam as inovações incrementais ou as inovações radicais. Destacamos ainda as duas perspectivas dos setores prestadores de serviços: aquela ligada aos serviços produtivos (manutenção de equipamentos, oferta de insumos, gestão para a otimização de processos e financiamento para a aquisição de produtos) e aquela que se relaciona com o consumo final de serviços (sejam exames ou informações que atinjam pacientes ou empresas médicas). Por sua vez, as indústrias produtoras de bens também prestam serviços produtivos, especialmente as grandes corporações, intensivas em tecnologia. No quadro também se mostra a chamada regulação híbrida (ANTAS JR., 2012), caracterizada pela ação, solidária ou contraditória, de Estado, corporações e organizações de solidariedade. Esses agentes da regulação híbrida concorrem para a compreensão do contexto político e institucional do complexo industrial da saúde, relacionando-se entre si: a) Estado, nos processos de regulação e promoção desse complexo; b) instituições de ciência e tecnologia, em função da intensidade de conhecimento empregada, "fonte essencial de inovações, que representam o fator crítico de competitividade dos segmentos do complexo" (GADELHA, 2003: 525); e c) sociedade civil, referente ao caráter social da produção em saúde, "certamente uma das atividades econômicas em que os grupos de interesse e as políticas públicas incidem de modo mais acentuado" (GADELHA, 2003:525).

Figura 1: Complexo industrial da saúde - dos circuitos espaciais produtivos da saúde ao consumo final de produtos e serviços. 


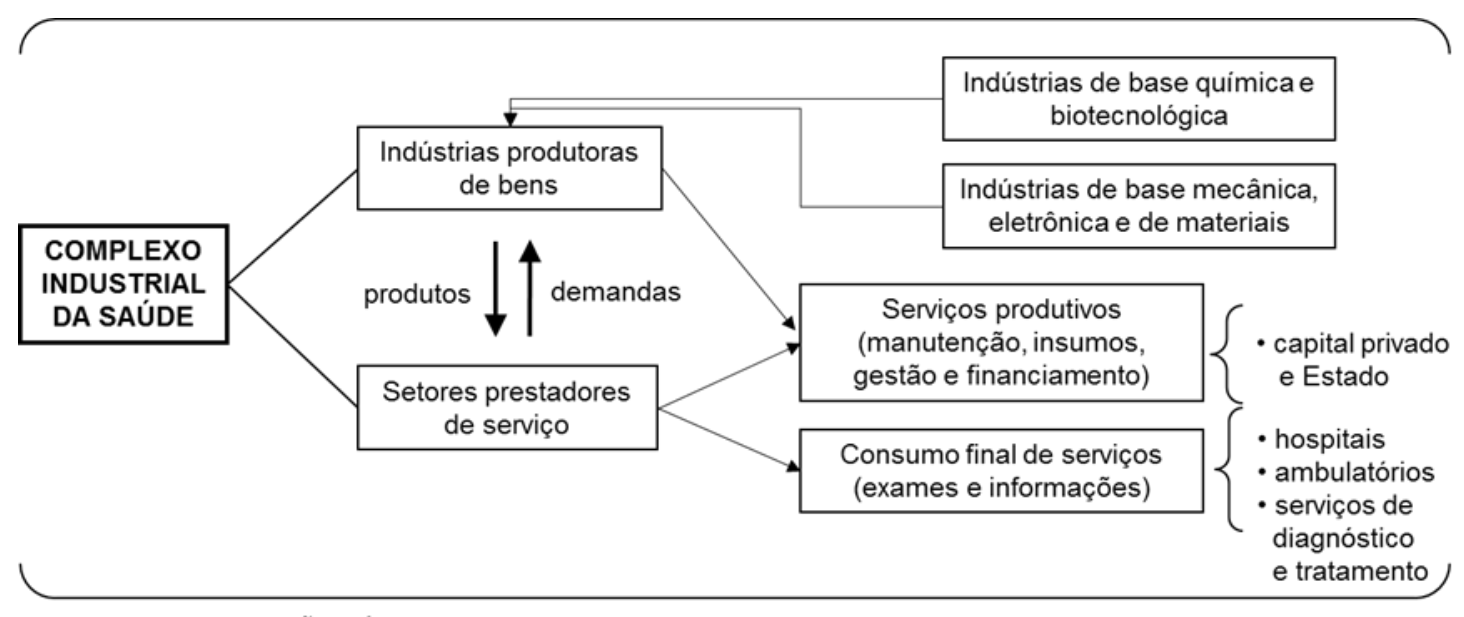

REGULAÇÃO HIBRRIDA: Estado, corporações hegemônicas e organizações de solidariedade

Fonte: SANTOS, 2016: 26.

A proposta do complexo parte do pressuposto de que, ao perceber uma demanda crescente por bens e serviços de saúde, há a oportunidade de ampliar o acesso à saúde e o desenvolvimento do país em setores de inovação, ciência e tecnologia (BERCOVICI, 2013). O reconhecimento e institucionalização do complexo industrial da saúde (12 de maio de 2008) no âmbito do Ministério da Saúde ${ }^{2}$ regulamenta uma política econômica e social. Em seu Artigo $1^{\circ}$, estabelece o GECIS - Grupo Executivo do Complexo Industrial da Saúde -, com o objetivo de "promover medidas e ações concretas visando à criação e implementação do marco regulatório brasileiro referente à estratégia de desenvolvimento do Governo Federal para a área da saúde, segundo as diretrizes das políticas nacionais de fortalecimento do complexo produtivo e de inovação em saúde" (BRASIL, 2008).

Assim, por Decreto, competiria ao GECIS desenvolver e implantar de forma integrada o marco regulatório para efetivar as estratégias e diretrizes da Política Industrial, Tecnológica e de Comércio Exterior brasileira, criando um ambiente favorável para o desenvolvimento do complexo industrial da saúde por meio de grupos de trabalho, sólida base de suporte com conhecimentos técnicos e especializados para essas atividades.

Suas diretrizes estabelecem como prioridades: o incentivo à produção e à inovação em saúde no país, ampliando a competitividade de todo o complexo no mercado interno e externo, simplificando e agilizando seus processos regulatórios e administrativos; a garantia de apoio à qualidade da produção nacional e isonomia na

\footnotetext{
${ }^{2}$ Decreto presidencial de Luiz Inácio Lula da Silva, assessorado por Jorge Gomes Temporão, Miguel Jorge, Sergio Machado Rezende e Dilma Rousseff.
} 
regulação sanitária; o apoio à criação de incentivos financeiros a áreas estratégicas ao fortalecimento do complexo; e, por fim, o estímulo ao uso do poder de compra do Sistema Único de Saúde para os produtos nacionais (BRASIL, 2008).

Posteriormente, novas leis e decretos foram estabelecidas, dando continuidade à sua estruturação e fortalecimento - a saber, o Decreto n. 7.540, de 2 de agosto de 2011, da Presidenta da República Dilma Rousseff (instituição do Plano Brasil Maior, com novas diretrizes para a política industrial, tecnológica e de comércio exterior), a Portaria n. 506, de 21 de março de 2012, do Ministério da Saúde (Programa para o Desenvolvimento do Complexo Industrial da Saúde - PROCIS) e o Decreto presidencial n. 8.065, de 7 de agosto de 2013 (estrutura organizacional do Ministério da Saúde, onde encontramos a Secretaria de Ciência, Tecnologia e Insumos Estratégicos central para a efetividade do complexo, comandada entre 2011 e 2015 por Carlos Augusto Grabois Gadelha ${ }^{3}$-, formada pelo Departamento de Assistência Farmacêutica e Insumos Estratégicos, pelos departamentos de Ciência e Tecnologia, do Complexo Industrial e Inovação em Saúde e, por fim, de Gestão e Incorporação de Tecnologias em Saúde).

Com o olhar orientado sobretudo por Gadelha (2006), verificamos que, no caso brasileiro, o complexo industrial da saúde incorporou à política industrial na área da saúde elementos do desenvolvimento das atividades econômicas, indicando os desafios de: articular a dinâmica industrial e de inovação com o sistema de assistência médica; constituir uma base de conhecimento endógena para a capacitação local, tácita e sistêmica; formar uma rede que envolva organizações de produção, pesquisa, financiamento e regulação; estimular arranjos produtivos localmente interligados em saúde; garantir a prospecção permanente de novas tecnologias; procurar montar sistemas regulatórios não tradicionais; e, por fim, permitir a introdução de mudanças institucionais no setor produtivo e nas instituições de suporte que respondam aos novos desafios contemporâneos. O autor destaca, ainda, a necessidade de olhar para a saúde de maneira ampla, não setorial, como uma oportunidade estratégica de desenvolvimento e de redução da dependência brasileira (GADELHA, 2006).

\footnotetext{
${ }^{3}$ Em janeiro de 2015, o economista Carlos Augusto Grabois Gadelha (que cunhou e difundiu o conceito de complexo industrial da saúde adotado aqui) deixou o cargo de secretário geral da Secretaria de Ciência, Tecnologia e Insumos Estratégicos (SCTIE) do Ministério da Saúde para assumir a secretaria de Desenvolvimento da Produção do Ministério do Desenvolvimento, Indústria e Comércio Exterior (MDIC). Em seu antigo posto assumiu o médico Jarbas Barbosa da Silva Júnior, que ali permaneceu entre janeiro e julho, até ser nomeado para o posto de diretor da Agência Nacional de Vigilância Sanitária (Anvisa). Atualmente, o cargo de secretário geral da SCTIE é ocupado pelo também médico Adriano Massuda, ex-Secretário de Saúde da cidade de Curitiba (2013-2015).
} 
Sem perder de vista o interesse social do setor, ou seja, a demanda social de bens e serviços que lhe é inerente, poderíamos acrescentar-lhe questões de ordem socioespacial, como: o aumento da especialização dos lugares; a consolidação de uma urbanização corporativa; a seletividade do território e a assimilação de novas técnicas em diferentes escalas pelos lugares; as implicações sociais dessas diferenças e a persistência das diferenças socioespaciais; entre outras.

\section{Os Circuitos espaciais produtivos da saúde e os círculos de cooperação no espaço}

O complexo industrial da saúde, então, é entendido pela coexistência de diversas indústrias de produtos variados, organizadas em circuitos que mobilizam: matériasprimas fornecidas por todo o mundo; substâncias primárias de alto conteúdo tecnológico e de produção rara e especializada; produtos semiacabados que serão finalizados conforme as leis ambientais e sanitárias nacionais; até produtos já finalizados (ANTAS JR., 2012). Assim, não temos no complexo industrial somente uma produção nacional, já que a maior parte dos produtos de alto valor agregado são caracterizados por apresentar alta tecnologia e conhecimento científico sendo necessário, portanto, a importação de produtos semimanufaturados de grandes corporações transnacionais ligadas à área de química fina, eletrônica, biomedicina e fármacos pelas indústrias nacionais, ou a importação de produtos acabados. É essa coexistência de agentes econômicos em cooperação que produz a complexidade atual da divisão territorial do trabalho permitindo caracterizá-lo como formador de circuitos espaciais produtivos da saúde (ANTAS JR., 2011a).

Em período de efetiva ação de uma lógica global, é preciso recorrer à noção de circuito espacial produtivo para entender a produção, a prestação de serviços e o consumo mundializado. Para existir, esses circuitos espaciais de produção estão pautados em uma rede de infraestruturas e em bases normativas regulatórias que padronizam a produção e os serviços (CASTILLO, 2008), dependentes de outros arranjos denominados círculos de cooperação no espaço (SANTOS, 2012; SILVEIRA, 2010; CASTILLO \& FREDERICO, 2010, ANTAS JR., 2015), dando uma visão 
dinâmica do modo como perpassam os fluxos (normas, ordens, informações) pelos territórios.

Temos no território brasileiro a formação de uma rede unindo a prestação de serviços de saúde e a produção industrial de todo tipo de produtos. A produção de artefatos com grau mais elevado de tecnologia está associada à aplicação de pesquisas stricto sensu, a instituições (públicas e privadas) capazes de conectar os agentes produtivos entre si e entre os serviços, ao papel regulatório que as instituições públicas exercem, e sobretudo, à circulação e à logística para que o transporte dos produtos seja feito de forma rápida, segura e flexível (ANTAS JR., 2011a).

Podemos dizer que os circuitos espaciais de produção pressupõem a circulação de matéria (fluxos materiais) no encadeamento das instâncias geograficamente separadas da produção, distribuição, troca e consumo de determinado produto, num movimento permanente; os círculos de cooperação no espaço, por sua vez, tratam da comunicação, consubstanciada na transferência de capitais, ordens, informação (fluxos imateriais), garantindo os níveis de organização necessários para articular lugares e agentes dispersos geograficamente, isto é, unificando, através de comandos centralizados, as diversas etapas, espacialmente segmentadas, da produção (CASTILLO \& FREDERICO, 2010: 464-465).

Os círculos de cooperação no espaço, capazes de conectar fluxos de capitais, ordens e informações organizando a produção que se dá segmentada pelo território, concentram muitos agentes que cooperam para a realização de determinado processo produtivo sendo possível observar a ação corporativa dessas empresas hegemônicas no território.

Portanto, reforçado por Antonio Carlos Moraes (1985), enquanto o circuito espacial produtivo pode ser entendido como a dimensão material da produção, distribuição, troca e consumo, os círculos de cooperação no espaço podem ser vistos como os fundamentos imateriais da divisão espacial da produção, superando as dependências e as oscilações das demandas locais ou regionais (ANTAS JR., 2014). Sintetizando associações, hierarquias e fluxos em conexão, se dão a partir de empresas e poderes públicos locais, regionais ou nacionais; entre empresas, associações não governamentais e instituições sem fins lucrativos; por financiamentos oferecidos por instituições bancárias; por parcerias com universidades, institutos de pesquisa e 
certificadoras de qualidade; com o trabalho de firmas de consultoria jurídica, de mercado e de publicidade; entre outros, "capazes de uma produção normativa fundamental para operarem os circuitos espaciais" (ANTAS JR., 2015: 2).

\section{Hospitais: a organicidade do complexo}

Diante do exposto percebemos que as inovações no setor saúde necessitam de grande intercâmbio com o setor científico e também com a prática médica, ou seja, as novas descobertas científicas influenciam as práticas médicas e, ao mesmo tempo, a própria prática gera um fluxo também importante de informações que necessitam ser melhor explicadas por pesquisas (ALBUQUERQUE, 2004). Nesse sentido, segundo Albuquerque (2004) torna-se imprescindível compreender três elementos: o complexo médico-industrial formulado por Cordeiro (1980 apud ALBUQUERQUE, 2004) com a interação entre universidades, assistência médica, indústrias; o sistema biomédico de inovação sugerido em um estudo de Hicks \& Katz (1996 apud ALBUQUERQUE, 2004) em que detectaram uma forte colaboração de hospitais da Inglaterra na produção científica daquele país; e as interações entre universidades e indústrias na produção de inovações médicas no estudo de Gleijns \& Rosenberg (1999 apud ALBUQUERQUE, 2004). Segundo Albquerque (2004), esses três pontos quando articulados possibilitam a visualização dos fluxos de informações, científicas e tecnológicas, no sistema de inovação da saúde atentando para as instituições/agentes participantes (ALBUQUERQUE, 2004) (Figura 2).

\section{Imagem 2}

Fluxo de informações científicas e tecnológicas no sistema de inovação do setor saúde: o caso de países com sistemas maduros. 


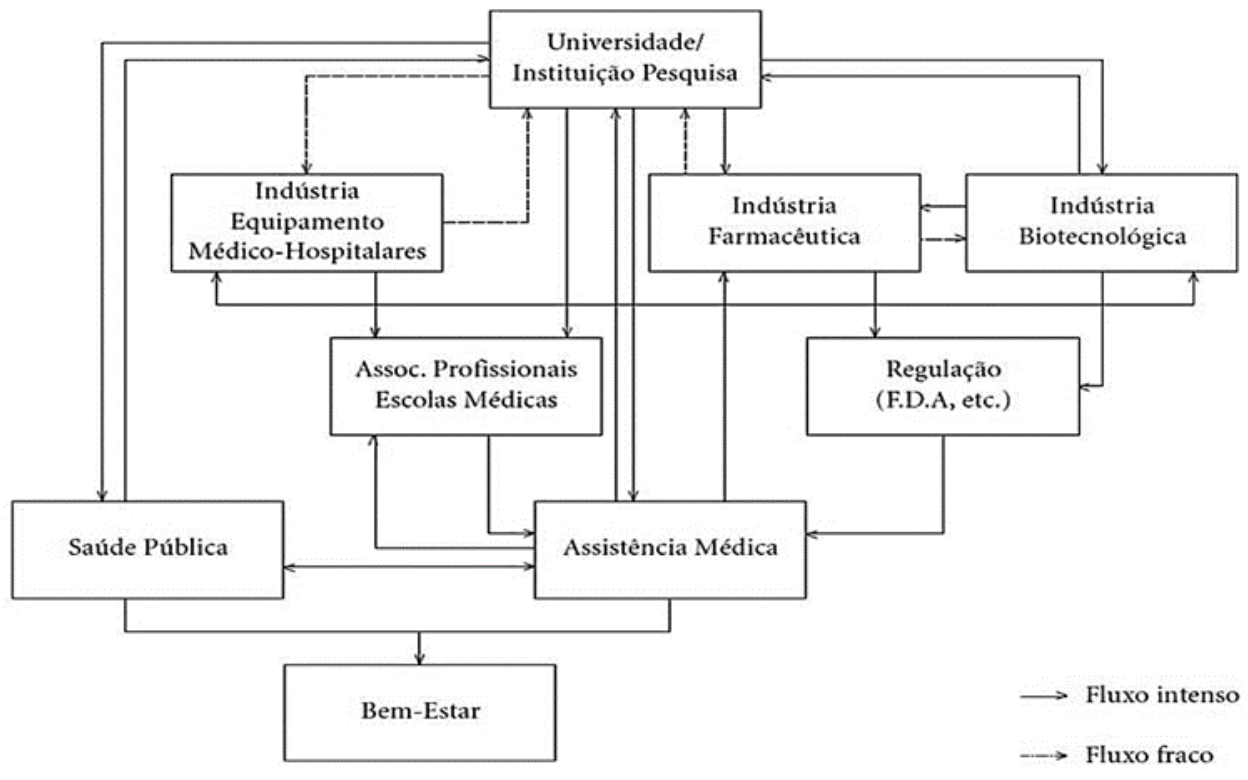

Fonte: Albuquerque, 2004: 284.

Nas universidades e instituições de pesquisa há a origem e a chegada de grande parte dos fluxos de informações científica e tecnológica, "se caracterizariam como um verdadeiro foco e centro de convergência de fluxos" (ALBUQUERQUE, 2004: 283). Os hospitais, assim como outras formas de assistência médica como clínicas e postos de saúde, também apresentam uma participação ativa no fluxo de informações com as indústrias e as universidades. Atentamos para o importante papel que os hospitais e centros médicos acadêmicos apresentam pela grande capacidade de implantação e difusão das inovações. As instituições de regulação, associações profissionais e escolas médicas participam do sistema como filtros das inovações universitárias e industriais tendo o médico como selecionador de novos tratamentos sem interesses mercantis (ao menos sob o viés social que a saúde apresenta). As indústrias apresentam diferentes graus de integração com as universidades podendo formar clusters, como o de biotecnologia gerado na Califórnia a partir do legado instituído pelo cluster de informática como infraestrutura, rede de comunicação, mão-de-obra, conexão com universidades aumentando a cooperação entre as atividades acadêmicas e científicas de profissionais de diferentes disciplinas e especialidades. A partir das interações entre universidades e instituições de pesquisa e com as indústrias, a saúde pública entra em ação pelo sistema de assistência médica com o propósito de levar bem-estar para a população por meio da efetividade das inovações. Vemos, portanto, o quanto o setor saúde é amplamente baseado numa ciência de pesquisas interdisciplinares, ou seja, dependente da formação de profissionais de diferentes áreas. E que nessa articulação 
entre industrias, universidades e instituições de pesquisa com a produção de inovações e bem-estar social temos os hospitais como pontos cruciais de integração simultânea a esses dois sistemas (inovações e bem-estar).

A partir da entrada cada vez maior do coeficiente tecnológico na medicina, a instituição hospitalar passou por profundas modificações tanto na forma de seu funcionamento quanto em sua estrutura, a fim de incorporar os novos materiais e equipamentos, ampliando o nexo existente entre os serviços e as indústrias culminando na instituição do complexo industrial da saúde no Ministério da Saúde (ANTAS JR., 2011a). Além de serem centrais por consumirem produtos industriais de alto e baixo valor agregado, também concentram trabalhos especializados não relacionados somente aos profissionais da saúde, como médicos, enfermeiros, fisioterapeutas, psicólogos, etc., mas também técnicos em informática, economistas, gestores hospitalares, técnicos de manutenção, etc., além de pesquisadores ligados a universidades (ANTAS JR., 2011a).

Com o desenvolvimento da técnica atrelada ao conhecimento científico, o paciente passa a ser visto de forma cada vez mais artificializada proporcionando novas possibilidades de intervenção sobre a doença, sendo os hospitais os centros de excelência no exercício dessa medicina moderna. Esse desenvolvimento técnicocientífico da clínica médica pautada em exames e no tratamento de doenças fez com que a medicina se tornasse uma atividade econômica, lugar de consumo de serviços de saúde, inserindo os hospitais na lógica urbana (DAVID, 2010).

Nessa proposta hospitalar estão inclusos diversos setores industriais - mecânica, óptica, eletrônica, química -, constituindo uma grande cadeia de relações com uma medicalização da saúde, ou seja, "processo histórico no qual a introdução crescente de inovações tecnológicas nas atividades em saúde tende a confundir o consumo de serviços médicos com a preservação da saúde, transformando os primeiros em demanda crescente para os serviços" (DAVID, 2010: 27), além de associar a eficiência médica a um maior coeficiente tecnológico.

Os hospitais geram uma gama enorme de demandas fazendo com que haja a coexistência de várias divisões territoriais do trabalho formadas por circuitos espaciais produtivos da saúde através da intensificação das trocas de equipamentos, materiais, informações, normas operando em diferentes lugares, o que reflete no processo de modernização dos hospitais e de serviços de saúde inseridos em um processo de mercantilização aumentando as necessidades de trocas regionais (DAVID, 2010). 
As formas geográficas são alocadas no espaço para que seja funcional à propagação dessas trocas regionais, possibilitando a instalação de diversos circuitos espaciais produtivos em um mesmo lugar, formando "um mosaico, no qual convivem formas com racionalidades e conteúdos diversos, concebidas e implantadas em momentos distintos" (CASTILLO \& FREDERICO, 2010: 463). O desenvolvimento técnico e informacional permite essa articulação entre áreas cada vez mais dispersas, daí a compreensão do conceito de círculos de cooperação apreendido como os fluxos informacionais que colocam em "contato" etapas da produção alocadas em diferentes lugares.

Desse modo, tanto o Estado - e o papel das universidades públicas aí é crucial - quanto as corporações acabam produzindo uma expansão urbana corporativa, condicionadora e também condicionada pela expansão do SUS, que investe fortemente em instalações públicas no interior do estado (complexos hospitalares, centros de pesquisa, universidades em cooperação com Estado e corporações) (ALMEIDA \& ANTAS JR., 2011: 3).

Os objetos ligados à saúde são em grande parte construídos e fornecidos por grandes corporações, desde as indústrias às prestadoras de serviços que exercem grande poder nas refuncionalizações dos espaços da cidade adaptando ou implementando novos objetos e novos sistemas de fornecimento e produção de insumos ao lado de transformações em outros ramos da economia urbana como transporte, habitação, educação (ANTAS JR., 2011b). Essas refuncionalizações refletem esse novo paradigma da medicina baseada na grande dependência de novas tecnologias e conhecimentos científicos, promovendo constantes mudanças nos padrões tecnológicos que levam a grandes impactos na transformação da cidade (ANTAS JR., 2011b).

Citamos como exemplo um eixo, localizado na cidade de São Paulo, que faz a ligação entre o Hospital das Clínicas/Faculdade de Medicina (USP) - bairro Cerqueira César - e o Hospital São Paulo/Escola Paulista de Medicina - bairro Vila Clementino -, passando pelas imediações da avenida Paulista. Esse eixo é formado por um adensamento de estabelecimentos e serviços de saúde de alta complexidade da rede pública e privada incluindo instituições de ensino e residência médica. É nessa lógica de consolidação e expansão do complexo industrial da saúde e de seus correspondentes circuitos espaciais produtivos que consideramos a grande importância dos hospitais 
desse eixo, sobretudo aqueles localizados em seus extremos, o Hospital das Clínicas/Faculdade de Medicina e o Hospital São Paulo/Escola Paulista de Medicina, como lugares de pesquisa e de absorção dessa produção que é levada para o consumo na forma de assistência médica, promovendo as devidas mudanças na cidade.

Esses complexos hospitalares de alta complexidade - Hospital das Clínicas e Hospital São Paulo - são os lugares onde há o aprofundamento do padrão tecnológico fortemente acelerado após a criação do Sistema Único de Saúde que possibilitou a incorporação dos novos conhecimentos científicos e tecnológicos no diagnóstico, tratamento e cura de doenças, além de proporcionar ao longo do tempo transformações na cidade pela inserção de novas funções, dotadas de novas tecnologias e informações, definidas por novas divisões do trabalho e exigentes de mudanças no espaço capazes de acolher essa nova lógica. Essas mudanças, no entanto, não se dão pela destruição do que já existe, mas por diferentes níveis de refuncionalizações de objetos e sistemas técnicos, a princípio de forma pontual com mudanças na circulação, na renda do solo urbano, no perfil de ocupação das classes alta e média, novos serviços, etc., (ANTAS JR, 2011b; SOUZA, 2006). Ao longo do tempo promovem as reestruturações da cidade capazes de manter nossa formação socioespacial na mundializada divisão social e territorial do trabalho (ANTAS JR., 2011b).

Embora Albuquerque (2004) afirme que no Brasil o papel que as universidades e institutos de pesquisa tem apresentado pouco impacto no sistema como um todo, predominando ainda a absorção de conhecimentos gerados no exterior sem o desenvolvimento de uma massa crítica que promova inovações brasileiras no setor saúde, destacamos a Universidade Federal de São Paulo, sobretudo a Escola Paulista de Medicina e o Hospital São Paulo e a Universidade de São Paulo e a Faculdade de Medicina e o Hospital das Clínicas por apresentarem grandes contribuições ao desenvolvimento científico, tecnológico e de prática médica para a medicina brasileira, por meio de investimentos na pesquisa, na compra de equipamentos, na melhor assistência médica, e na parceria com indústrias:

- A Universidade Federal de São Paulo possui 95 grupos que agregam 49,2\% dos pesquisadores e 54,5\% dos doutores pesquisadores da capital (exemplo: Centro Paulista de Pesquisa em HIV/Aids, Centro de Doenças Respiratórias Crônicas e Centro de Hipertensão Arterial) (SEADE, 2010). Apresentam também, a Escola Paulista de Medicina e Hospital São Paulo, um Centro de Pesquisas Clínicas e Cirúrgicas "Oswaldo 
Ramos" criado em 1997 com 36 laboratórios e centros de estudos com 16 linhas de pesquisa. Em 2005, criou-se a Fundação de Apoio à Universidade Federal de São Paulo para amparar a universidade em projetos de ensino, pesquisa, extensão, inovação e transferência de conhecimento.

- Por sua vez, a Universidade de São Paulo apresenta 47 grupos de pesquisa somando $24,5 \%$ dos recursos humanos e 24,2\% dos pesquisadores doutores da cidade, além de deter $33 \%$ da produção técnico-científica e $29 \%$ dos artigos publicados em periódicos especializados internacionais (SEADE, 2010). Com mais de 200 grupos de pesquisa com a associação de docentes, médicos do $\mathrm{HC}$, alunos de graduação e pós-graduação e com financiamentos que corresponderam no período entre 2007-2010 a aproximadamente R\$ 210 milhões (parte da verba destinada pelo Sistema Único de Saúde para o Hospital das Clínicas/Faculdade de Medicina USP e recursos de órgãos governamentais, organizações nacionais e internacionais, agências de fomento internacionais, empresas privadas e fundações) realizam estudos sobre as doenças mais comuns no país, as aplicações dos conhecimentos produzidos pela tecnologia moderna (Genômica e Imunologia), as epidemias, as vacinas, os impactos das poluições ambientais, as novas técnicas de cirurgia, transplantes e terapia celular auxiliando políticas públicas (CAVALLIERI et. al., 2012). O Sistema FMUSP/HC compõem hoje o mais importante centro de pesquisa brasileiro, no qual os Laboratórios de Investigação Médica (LIMs) contribuíram com a publicação, em 2009, de 1.293 artigos em periódicos indexados no Institute for Scientific Information na área de saúde, ou seja, “4\% da publicação brasileira e 2\% da publicação latino-americana (todas as áreas do conhecimento)" (CAVALLIERI et. al., 2012: 163). Outro apoio significativo à pesquisa e à saúde, além dos LIMs, é a Fundação Faculdade de Medicina criada em 1986 pela Associação dos Antigos Alunos da Faculdade de Medicina da Universidade de São Paulo, sendo uma instituição de direito privado e sem fins lucrativos que apoia o ensino, a pesquisa e a assistência médica da Faculdade de Medicina e do Hospital das Clínicas. Atua no gerenciamento financeiro, dos recursos humanos, de compras, etc. e na formação de parcerias com outras instituições. 


\section{Considerações finais}

Nas últimas décadas houve, de um lado a criação do Sistema Único de Saúde com um arcabouço técnico e normativo, e por outro lado uma ampliação do raio de ação de empresas ligadas aos serviços de saúde (laboratórios farmacêuticos, laboratórios de análises clínicas, empresas de planos de saúde, clínicas particulares, produtores e fornecedores de equipamentos médicos) aumentando a dependência por tecnologia e por recursos de terceiros (ALMEIDA \& BICUDO, 2010). Assim, configura-se também sua face perversa, pois, para que a saúde atenda às necessidades do mercado, é preciso criar constantemente novas necessidades, novos medos, novas dúvidas. O que faz bem e o que faz mal passa a ser mediado por interesses mercantis e pela garantia de lucros. Esse é o poder que as indústrias hegemônicas alcançaram pelo domínio da técnica, da ciência e da informação. Fabricam objetos técnicos médicos duros e eficientes, capazes de determinar o modo como o trabalho será dividido, inclusive em termos da forma organizacional e da regulação (DAVID, 2010).

Nessa perspectiva, a compreensão da chamada medicina tecnológica subsidia o contexto em que se inserem os hospitais: como vimos, esse paradigma é pautado na interdependência e na cooperação, com o largo emprego de medicamentos e de instrumentos diagnósticos intensivos em tecnologia, orientadores das atuais concepções de medicina, trabalho médico e cura.

O hospital, sistema técnico-científico cada vez mais informatizado, é objeto central para a produção industrial e científica dos circuitos espaciais produtivos da saúde, círculos de cooperação no espaço e para o complexo industrial da saúde. Isso pode ser visto na inseparabilidade entre a produção industrial de diversos tipos de insumos - com alto conhecimento aplicado e pesquisa stricto sensu associadas a instituições públicas e privadas e com uma logística que possibilita o transporte seguro dos produtos - e os serviços de saúde que funcionam como pontos de convergência de interesses corporativos, pela intensificação das trocas de equipamentos, materiais, informações e normas produzidos em diferentes lugares e transportados pelas redes. A inovação faz parte desse processo.

O estudo científico está estreitamente ligado à área da saúde, numa rede de fluxos de informações que podem ter como origem ou destino a prática clínica, a indústria ou centros de pesquisas (privados, públicos, acadêmicos e/ou empresariais). 
Podemos dizer que a medicina moderna está estruturalmente assentada em três pilares: técnica, ciência e informação. Isso implica objetos cada vez mais funcionais e eficientes exigindo a constituição de famílias de objetos e sistemas técnicos para funcionarem plenamente. $\mathrm{Na}$ medicina, essa relação entre técnicas sistêmicas e especializadas e a possibilidade da universalização das tecnologias resultou em procedimentos especializados para cada necessidade, que podem ser feitos em vários lugares do mundo.

\section{Referências Bibliográficas}

ALBUQUERQUE, Eduardo. M. et al (2004). Pesquisa e inovação em saúde: uma discussão a partir da literatura sobre economia da tecnologia. Ciência \& Saúde Coletiva. Brasil, vol. 9, n. 2, pp. 277-294.

ALMEIDA, Elisa. P. \& ANTAS Jr., Ricardo. M (2011). Os serviços de saúde no estado de São Paulo - seletividades geográficas e fragmentação territorial. In: MARINHO, Gabriela. S. M. C. \& MOTA, André. (Org.). Práticas Médicas e de Saúde nos Municípios paulistas: a história e suas interfaces. São Paulo: Museu Histórico Prof. Carlos da Silva Lacaz.

ALMEIDA, Elisa. P. \& BICUDO, Edison (2010). Psicoesfera e medicina: meio construído urbano e congressos médicos na América Latina. Revista Geográfica Venezolana. Venezuela, vol. 51, n. 2, pp. 179-201.

ANTAS JR., Ricardo. M (2015). Complexos industriais, circuitos espaciais produtivos e direito reflexivo. In: Encontro Nacional da Associação Nacional de PósGraduação e Pesquisa em Planejamento Urbano e Regional. Belo Horizonte, pp. $1-16$.

(2014). O complexo industrial da saúde no Brasil: uma abordagem a partir dos conceitos de circuito espacial produtivo e círculos de cooperação no espaço. GEOgraphia. Brasil, vol. 16, n. 32, pp. 38-67.

(2012). Considerações sobre agentes relevantes nos círculos de cooperação do complexo industrial da saúde no território brasileiro. In: Encuentro de Geógrafos de América Latina. Peru, pp. 75-89.

(2011a). Circuitos espaciais produtivos da saúde, serviços médico-hospitalares e transformações da urbanização no território paulista. In: IX Enanpege - Encontro Nacional da Associação Nacional de Pós-Graduação e Pesquisa em Geografia, pp. 1-8.

(2011b). Notas sobre o uso do conceito de Circuitos Espaciais Produtivos para estabelecer o nexo entre a Reestruturação Urbana e as Refuncionalizações do Espaço: um estudo sobre os fixos de saúde no Estado de São Paulo. In: Anais do XIV Encontro Nacional da ANPUR. Rio de Janeiro, pp. 1-15.

BERCOVICI, Gilberto (2013). Complexo industrial da saúde: desenvolvimento e proteção constitucional ao mercado interno. Revista de Direito Sanitário. Brasil, vol. 14, n. 2, pp. 9-42.

BRASIL (2008). Decreto de 12 de maio de 2008. Cria, no âmbito do Ministério da Saúde, o Grupo Executivo do Complexo Industrial da Saúde - GECIS, e dá outras providências. Disponível em: <http://www.planalto.gov.br/ccivil_03/_Ato2007- 
010/2008/Dnn/Dnn11578.htm>. Acesso em: 8 out. 2013.

(2012). Portaria n. 506, de 21 de março de 2012. Institui o Programa para o Desenvolvimento do Complexo Industrial da Saúde - PROCIS e seu Comitê Gestor. Disponível em: <http://bvsms.saude.gov.br/bvs/saudelegis/gm/2012/pr t0506_21_03_2012.html>. Acesso em: 8 out. 2013.

(2011). Decreto n. 7.540, de 2 de agosto de 2011. Institui o Plano Brasil Maior PBM e cria o seu Sistema de Gestão. Disponível em: <http://www.planalto.gov.br/ccivil_03/_Ato2007-

2010/2008/Dnn/Dnn11578.htm>. Acesso em: 8 out. 2013.

CASTILLO, Ricardo. (2008). Sustentabilidade, globalização e desenvolvimento. In: OLIVEIRA, Márcio. P. et al. (Org.). O Brasil, a América Latina e o mundo. Rio de Janeiro: ANPEGE.

\& FREDERICO, Samuel. (2010). Espaço geográfico, produção e movimento: uma reflexão sobre o conceito de circuito espacial produtivo. Sociedade \& Natureza. Brasil, vol. 22, n. 3, pp. 461-474.

CAVALLIERI, Angela. C.; et. al (2012). Os Laboratórios de Investigação Médica do Hospital das Clínicas da FMUSP (LIMs). In: MARINHO, Gabriela. S. M. C. \& MOTA, André. (org.). Da Faculdade de Medicina e Cirurgia de São Paulo à Faculdade de Medicina da Universidade de São Paulo: conjunturas e contextos. São Paulo: CD.G Casa de Soluções e Editora.

DAVID, Virna. C. (2010). Território usado e circuito superior marginal: equipamentos médico-hospitalares em Campinas, Ribeirão Preto e São José do Rio Preto (SP). Dissertação (Mestrado em Geografia Humana). Faculdade de Filosofia, Letras e Ciências Humanas, Universidade de São Paulo, São Paulo.

GADELHA, Carlos. A. G. (2006). Desenvolvimento, complexo industrial da saúde e política industrial. Revista de Saúde Pública, São Paulo, n. 40, p. 11-23.

(2003). O complexo industrial da saúde e a necessidade de um enfoque dinâmico na economia da saúde. Ciência \& Saúde Coletiva, Rio de Janeiro, vol. 8, n. 2, p. 521-535.

\& QUENTAL, Cristiane. \& FIALHO, Beatriz. C. (2003). Saúde e inovação: uma abordagem sistêmica das indústrias da saúde. Cadernos de Saúde Pública. Rio de Janeiro, vol. 19, n. 1, p. 47-59.

MORAES, Antonio. C. R. (1985). Os circuitos espaciais de produção e os círculos de cooperação no espaço. Departamento de Geografia, Faculdade de Filosofia, Letras e Ciências Humanas, Universidade de São Paulo. Mimeo.

MOTA, André. (2005). Tropeços da medicina bandeirante: medicina paulista entre 1892-1920. São Paulo: EDUSP.

PAIM, Jairnilson. S. (2009). O que é o SUS. Rio de Janeiro: Editora Fiocruz.

SANTOS, Fernando. D. A (2016). O circuito espacial produtivo e a topologia dos equipamentos de radiologia e diagnóstico por imagem no território brasileiro. Dissertação (Mestrado em Geografia Humana). Universidade de São Paulo, São Paulo.

SANTOS, Milton. (2012). Metamorfoses do espaço habitado: fundamentos teóricos e metodológicos da geografia. São Paulo: Edusp.

SANTOS, Milton. \& SILVEIRA, María. L. (2001). O Brasil: território e sociedade no início do século XXI. Rio de Janeiro/São Paulo: Record.

SCHRAIBER, Lilia. B. (2008). O médico e suas interações: a crise dos vínculos de confiança. São Paulo: Hucitec.

(1993). O médico e seu trabalho: limites da liberdade. São Paulo: Hucitec. 
SEADE - FUNDAÇÃO SISTEMA ESTADUAL DE ANÁLISE DE DADOS (2010). Ciências da Vida Humana na Cidade de São Paulo. São Paulo.

SILVEIRA, María. L. (2010). Região e globalização: pensando um esquema de análise. Redes, Santa Cruz do Sul, vol. 15, n. 1, p. 74-88, jan./abr.

SOUZA, Marcelo. L. de (2006). A prisão e a ágora: reflexões em torno da democratização do planejamento e da gestão das cidades. Rio de Janeiro: Bertrand Brasil.

VIANA, Ana. L. A. \& SILVA, Hudson. P. \& ELIAS, Paulo. E. M. (2005). Economia política da saúde: introduzindo o debate. In: Anais da Jornada de Economia da Saúde. Disponível em: <http://abresbrasil.org.br/trabalhos/economia-politica-dasaude-introduzindo-o-debate.html>. Acesso em: 25 mai.2015.

Artigo recebido em 31 de janeiro de 2016.

Aprovado em 20 de março de 2016.

DOI: $10.12957 /$ intellectus.2016.23840 